\title{
Migration et emploi urbain : le cas de Ouagadougou au Burkina Faso
}

\author{
Zourkaléini Younoussi \\ UERD \\ Université de Ouagadougou \\ Burkina Faso
}

\author{
Victor Piché \\ Université de Montréal \\ Canada
}

\begin{abstract}
Résumé
L'objectif de la présente étude est d'examiner le lien entre migration et emploi dans un contexte urbain, celui de Ouagadougou la capitale du Burkina Faso. Nous privilégions ici l'accès au premier emploi rémunéré car il représente une étape cruciale dans le cheminement professionnel et constitue un indicateur-clé de la possibilité de se soustraire à la pauvreté. En corollaire, un accès tardif à un premier emploi rémunéré pourrait être source de pauvreté. A partir des données de l'enquête nationale, sur "dynamique migratoire, insertion urbaine et environnement au Burkina Faso ", menée en 2000, deux outils essentiels de l'analyse des biographies, les courbes de survie de Kaplan-Meier et les régressions semi-paramétriques à risques proportionnels, sont utilisés pour évaluer le temps nécessaire pour obtenir un premier emploi ou pour mieux comprendre ce qui mène au premier emploi rémunéré. Les caractéristiques individuelles (sexe, niveau d'instruction), et les informations recueillie sur l'origine familiale (l'activité du père et de la mère), et l'ethnie sont utilisées comme variables de contrôle. Les analyses présentées suggèrent que le rôle de la migration sur l'accès à un premier l'emploi joue très peu pour les femmes alors que les migrants semblent avoir un avantage net sur les non migrants. L'origine sociale semble avoir peu d'impact sur l'accès au premier emploi mais la crise économique et/ou et la mise en œuvre des programmes d'ajustement structurel font que la pauvreté touche plus les jeunes générations qui semblent accéder plus tardivement à un premier emploi rémunéré.
\end{abstract}

\section{Introduction}

Les données de deux enquêtes sur les conditions de vie des ménages, réalisées en 1994 et 1998, montrent que le niveau de la pauvreté reste élevé au Burkina Faso. En effet, la proportion de la population burkinabé vivant en dessous du seuil ${ }^{1}$ de pauvreté est estimée à $44,5 \%$ en 1994 et à $45,3 \%$ en 1998 (Burkina Faso, 2000 ; Somda et Sawadogo, 2001). Cette pauvreté demeure un phénomène essentiellement rural car, en 1998 par exemple, 94\% des pauvres sont du milieu rural contre seulement $6 \%$ en milieu urbain. Ouagadougou, la capitale, et Bobo-Dioulasso, deuxième ville du pays, affichent en 1998 les plus faibles pourcentages de pauvreté urbaine, soit $11,2 \%$, contre $25 \%$ pour les autres villes (INSD, 1999). Un tel contexte de disparité selon le milieu de résidence conforte la thèse économiste de la migration rurale-urbaine comme stratégie de lutte contre la pauvreté (Todaro, 1971, Potts, 2000). On peut donc supposer que plusieurs Burkinabé se voient obligés de quitter les zones pauvres (rural et autres villes) pour avoir accès aux opportunités d'emploi et de revenus disponibles dans les deux grandes villes du pays.

1 En 1998 le seuil de pauvreté est de 82672F CFA par adulte et par an alors qu'en 1994 , actualisé en coûts réels aux prix de 1998, il était de 72690F CFA 
Toutefois, comme dans plusieurs autres villes africaines (ILO, 1997, Antoine et Piché, 1998, Potts, 2000), les deux grandes villes du Burkina ont connu dans les années 90 des crises économiques liées, entre autres facteurs, aux programmes d'ajustement structurel. Par exemple, au cours de la période 1994-1998, le niveau de pauvreté est resté stable en milieu rural alors qu'il est passé de 7,8\% à 11,2 dans les deux grandes villes (Ouagadougou et Bobo) et de $18,1 \%$ à $25 \%$ dans les autres villes. La croissance de la pauvreté demeure donc un phénomène essentiellement urbain, laissant croire que la migration vers les villes, incluse dans les stratégies de survie des populations pauvres, pourrait ne plus jouer plus son rôle de soupape. En effet, face à une pauvreté urbaine croissante, la migration vers ces deux villes pourrait être interprétée comme participant à la marginalisation des migrants, particulièrement vulnérable sur un marché de travail perçu comme étant déjà limité, voire saturé. Dans un tel contexte on pourra s'attendre à ce que la pauvreté touche plus les jeunes générations et en particulier les jeunes migrants qui, du reste, auront probablement plus de difficulté à accéder à un emploi rémunéré.

On peut alors se demander ce qui explique l'afflux massif et constant des migrants vers la ville si la seule perspective est le chômage et la pauvreté extrême (Piché et Gingras, 1998). La littérature sur les liens entre migration et emploi met en opposition deux hypothèses concernant les performances économiques des migrants comparées à celles des non migrants (Piché, Mariko et Gingras, 1995; Kouamé, 2000). La première insiste sur les difficultés des migrants à avoir accès à un emploi urbain et leur faible potentiel d'insertion économique: ceux-ci viendraient en quelque sorte renflouer les rangs des sans emploi et des marginaux (. Adepoju, 1988; Todaro, 1997). La deuxième hypothèse suggère au contraire un accès plus facile des migrants à l'emploi urbain, hypothèse vérifiée par une série d'enquêtes rétrospectives dans plusieurs villes africaines (Piché et Gingras, 1998 et Bocquier et LeGrand, 1998 pour Dakar et Bamako²). En fait, de nombreux travaux récents ont montré à quel point les migrants et migrantes développent rapidement des capacités d'adaptation en milieu urbain, en particulier par la prolifération de petites entreprises informelles (Kouamé, 1991, Portes et Shauffer, 1993). Avec la crise de l'emploi en milieu urbain et l'accroissement des migrations de retour vers le milieu rural (Beauchemin, 2001 ; Pott, 2000), on peut se demander si la stratégie migratoire demeure encore viable.

L'objectif de la présente recherche est d'examiner le lien entre migration et emploi dans un contexte urbain, celui de Ouagadougou. Nous privilégions ici l'accès au premier emploi rémunéré car il représente une étape cruciale dans le cheminement professionnel et constitue un indicateur-clé de la possibilité de se soustraire à la pauvreté. En corollaire, un accès tardif à un

2 Kishimba (2002: 208) trouve des résultats contraires pour Yaoundé, les migrants (définis ici par le lieu de naissance) obtenant plus tardivement que les natifs leur premier emploi. 
premier emploi rémunéré pourrait être source de pauvreté. La comparaison entre migrants et non migrants permet de répondre à une première question, à savoir, la migration est-elle un atout pour l'emploi ? Dans le contexte de l'augmentation de la pauvreté urbaine, on pourrait s'attendre à ce que les migrants accèdent plus tardivement à l'emploi, surtout les plus jeunes générations.

Dans la plupart des enquêtes urbaines effectuées jusqu'à maintenant, il est difficile de répondre de façon concluante à ces questions étant donné les biais liés à la sélectivité migratoire. En effet, les résultats ne concernent que des échantillons de migrants et de migrantes enquêtés en milieu urbain, ignorant ceux et celles qui ont quitté après un séjour en ville. Si les caractéristiques de ces personnes non présentes au moment de l'enquête sont différentes de celles qui sont restées, les résultats sont biaisés dans un sens ou un autre, c'est-à-dire qu'ils sur-estiment la performance économique si les absents ont quitté parce qu'ils ont eu de la difficulté à trouver un emploi et ils la sous-estiment si au contraire ce sont les plus performants qui ont quitté. ${ }^{3}$ L'enquête dont nous nous servons ici étant une enquête nationale, nous sommes en mesure de réintégrer dans l'analyse les épisodes de séjour à Ouagadougou des personnes enquêtées ailleurs au Burkina ${ }^{4}$. C'est là une première différence notable avec les analyses de ce genre effectuées jusqu'ici.

Pour bien mesurer l'effet "net» de l'expérience migratoire, nous retenons un certain nombre d'autres facteurs reconnus comme importants dans l'étude de l'insertion économique (Antoine et Piché, 1998). Outre les effets de génération, qui nous permettent d'examiner l'évolution dans le temps, nous retenons la variable sexe, une variable de capital humain (l'éducation), deux variables d'origine sociale (activité du père et de la mère) et une variable culturelle (ethnie). ${ }^{5}$

\section{Méthodologie}

Cette étude est réalisée à partir des données de l'enquête nationale, sur « migration, insertion urbaine et environnement au Burkina Faso » menée en 2000 (Poirier et al., 2001). Comme on peut le constater au tableau 1, elle a porté sur 8644 biographies migratoires (dont 2838 dans la ville de Ouagadougou), recueillies auprès de 3517 ménages $^{6}$ (dont 1184 dans la ville

3 Nos analyses en cours semblent indiquer que c'est la deuxième hypothèse qui prévaut, i.e. ce sont les plus performants qui seraient partis.

4 Certes, nous ignorons toujours l'expérience des personnes ayant vécu à Ouagadougou et vivant à l'étranger au moment de l'enquête. Tout porte à croire que l'hypothèse de la sur-performance de ces migrants s'applique également à elles.

5 Sur la pertinence de l'origine ethnique, voir Traoré, 1997.

6 Cette enquête a été réalisée par l'Unité d'Enseignement et de Recherche en Démographie (UERD) de l'Université de Ouagadougou en partenariat avec le Centre d'Etudes et de Recherche sur la Population pour le Développement (CERPOD) et le Département de Démographie de l'Université de Montréal. 
de Ouagadougou). Nous utilisons les informations recueillies sur la vie active, soit les questions des modules deux et trois pour tenir compte du type d'activité économique exercée sur 3 mois ou plus et du lieu où cette activité à été exercée. Soulignons que les périodes d'inactivité liées aux périodes d'études, de maladie, de retraite et "au foyer " sont spécifiées au même titre que les périodes de travail ou de chômage. La vie active de l'individu se résume en une succession de périodes d'activité et/ou d'inactivité. Cette base de données se singularise des enquêtes urbaines déjà réalisées par la possibilité de prendre en compte les épisodes de passage à Ouagadougou de toutes les personnes recensées ailleurs dans le pays au moment de l'enquête. Par ailleurs, un effort particulier a été consenti pour mieux mesurer le travail des femmes, habituellement sous-estimé, ce qui rend difficile les comparaisons avec les autres enquêtes.

Tableau 1: Répartition de la population enquêtée selon la strate

\begin{tabular}{lrrrrrrrrr}
\hline \multirow{2}{*}{ Nombre } & \multicolumn{10}{c}{ Strate } \\
\cline { 2 - 8 } & Ouag. & Bobo & Banfora & Sahel & Est & Centre & Ouest & $\begin{array}{r}\text { Sud- } \\
\text { Ouest }\end{array}$ & Total \\
\hline Personnes & 6531 & 1734 & 2292 & 2616 & 2477 & 2683 & 2357 & 2309 & 22999 \\
Ménages & 1184 & 312 & 367 & 369 & 323 & 300 & 348 & 314 & 3517 \\
Biographies & 2838 & 691 & 879 & 931 & 784 & 921 & 810 & 790 & 8644 \\
\hline
\end{tabular}

Source : Rapport sur les objectifs, la méthodologie et le déroulement de la collecte (2002)

L'insertion sur le marché du travail rémunéré à Ouagadougou est notre variable dépendante et elle est appréhendée de deux façons: la rapidité d'entrée dans la vie active et les déterminants des chances d'accès à un premier emploi. Le groupe cible est la population sans emploi rémunéré à partir de l'âge de 12 ans. Le premier emploi rémunéré est défini comme étant la première occupation principale, ayant duré au moins trois mois. Ainsi, les périodes d'études, de maladie, de retraite, de chômage ou de foyer sont exclues au même titre que les activités d'apprentissage ou d'aide familial sans rémunération.

L'accès au premier emploi principal est basé sur l'examen des probabilités cumulées, ou encore l'intensité de connaître l'entrée en première activité au bout d'un certain nombre d'années de présence dans la ville de Ouagadougou. Le nombre d'années de présence est représenté chez les migrants par le nombre d'années d'attente vécues dans la ville avant l'acquisition d'un premier emploi rémunéré. Ainsi, toutes les périodes de séjour à Ouagadougou sont prises en compte jusqu'à l'obtention du premier emploi rémunéré (ou le départ) ; nous ne tenons pas compte du temps vécu hors de Ouagadougou. Pour les non-migrants, ce nombre d'années est plutôt égal à la différence entre l'âge d'entrée en première activité et l'âge de 12 ans. 
Les employés du secteur formel sont définis comme étant ceux qui reçoivent régulièrement des bulletins de salaires (une bonne indication des employeurs se conformant aux législations sociales et fiscales). Cette définition est conforme aux travaux récents qui définissent les activités informelles comme des activités génératrices de revenus et non réglementées par l'Etat (Bocquier et LeGrand, 1998 ; Piché et Gingras, 1998). La définition des différents statuts dans l'emploi se présente comme suit :

Indépendant : toute personne qui travaille pour son propre compte dans une entreprise individuelle. Elle n'emploie aucun salarié mais peut être aidée par des aides familiaux ou des apprentis non rémunérés ou par des manœuvres de façon temporaire (les marabouts, les prêtres, les pasteurs, etc. sont considérés comme des indépendants).

Travailleur à la tâche : toute personne qui est payée en fonction d'un travail déterminé et ponctuel. Elle ne touche pas un salaire régulier.

Salarié: tout individu touchant un salaire régulier de la part d'un employeur public ou privé en contrepartie du travail effectué. Un apprenti qui est rémunéré est considéré comme salarié.

Apprenti : toute personne qui apprend un métier sans recevoir de salaire.

Aide familial : toute personne qui travaille pour le compte d'une entreprise ou d'une exploitation appartenant à un membre de sa famille ou au ménage dont il fait partie. L'aide familial ne reçoit pas de salaire, mais en contrepartie il est logé et nourri.

Employeur : toute personne qui exploite sa propre entreprise ou qui exerce pour son propre compte une profession ou un métier et qui emploie au moins un salarié. (Étant donné les petits nombres, nous avons regroupé les employeurs et les indépendants.)

Pour tenir compte des problèmes d'effectifs nous avons retenu quatre générations: 1936-1955; 1956-1965; 1966-1975; 1976-1985. Le statut migratoire comprend deux catégories :

- Les non-migrants composés, d'une part, des personnes nées à Ouagadougou n'ayant jamais changé de départements et présentes à Ouagadougou au moment de l'enquête et, d'autre part, des personnes qui sont arrivées avant 12 ans et qui sont toujours présentes à Ouagadougou jusqu'au moment de l'enquête ;

- Les migrants composés des personnes ayant effectué au moins un changement de département après 12 ans et avec au moins une résidence à Ouagadougou, recensées ou non à Ouagadougou au moment de l'enquête. Dans l'analyse multivariée, nous distinguons les migrants par le dernier milieu de résidence avant l'obtention du premier emploi rémunéré à Ouagadougou. Dans les cas de troncature du fait de 
la date de l'enquête, il s'agira du milieu de résidence antérieur à celui de la date de l'enquête (pour les présents à Ouagadougou) ou du milieu de résidence antérieur au dernier séjour à Ouagadougou (pour les absents de Ouagadougou). Trois milieux de provenance sont pris en considération: rural, autres villes et étrangers.

Deux outils essentiels de l'analyse des biographies, les courbes de survie de Kaplan-Meier et les régressions semi-paramétriques à risques proportionnels, sont utilisés pour évaluer le temps nécessaire pour obtenir un premier emploi ou pour mieux comprendre ce qui mène au premier emploi rémunéré. Les caractéristiques individuelles (sexe, niveau $\mathrm{d}^{\prime}$ instruction), et les informations recueillies sur l'origine familiale (l'activité du père et de la mère), et l'ethnie sont utilisées comme variables de contrôle. L'introduction de ces variables nous permet de mettre en évidence les effets nets du statut migratoire et de la génération, au-delà des effets dus aux différences de marchés du travail et de qualifications.

\section{Résultats}

\section{Le marché du travail au moment de l'enquête}

La situation de l'emploi au moment de l'enquête est une première indication de la performance des migrants et des non migrants sur le marché du travail. Trois indicateurs du moment sont retenus ici : le chômage, le statut dans l'activité principale et le secteur d'activité. S'agissant du chômage (tableau 2), on constate comme cela est souvent le cas dans les villes africaines, qu'il est très faible pour tout le monde quoique légèrement plus élevé chez les hommes. En fait, la notion de chômage a peu de pertinence dans des situations où la législation sociale (e.g. assurance chômage) est inexistante pour la vaste majorité de la population (Pott, 2000). Ceci dit, en comparaison avec Dakar en 1989 et Bamako en 1992 où les proportions de chômage étaient plus élevées (respectivement de $15 \%$ et $10 \%$ pour les hommes et $4 \%$ et $5 \%$ chez les femmes; Bocquier et LeGrand, 1998), les niveaux estimés ici semblent particulièrement faibles. Outre les différences de dates des enquêtes, cette différence peut s'expliquer entre autres par un sous enregistrement des chômeurs. Par exemple, l'instruction suivante a été donnée aux enquêteurs : "lorsqu'une personne se déclare "chômeuse " et mène une activité ou plusieurs activités secondaires, on doit la considérer comme « au travail »(manuel de l'enquêteur, page 11)». Enfin, notons que les différences entre migrants et non-migrants, tout en étant faibles, montrent un léger avantage pour les migrants et les migrantes. 
Tableau 2 : Proportion de chômeurs au moment de l'enquête selon la génération, le sexe et le statut migratoire

\begin{tabular}{lcccc}
\hline \multirow{2}{*}{ Génération } & \multicolumn{2}{c}{ Sexe masculin } & \multicolumn{2}{c}{ Sexe féminin } \\
\cline { 2 - 5 } & Migrants & Non-migrants & Migrantes & Non-migrantes \\
\hline $1936-1955$ & 2,86 & 8,44 & 0 & 0 \\
$1956-1965$ & 3,71 & 0,33 & 0,82 & 0 \\
$1966-1975$ & 5,13 & 8,35 & 0 & 0,56 \\
$1976-1985$ & 5,16 & 4,28 & 0,7 & 1,94 \\
Total & 4,21 & 5,26 & 0,38 & 1,06 \\
\hline
\end{tabular}

En examinant le statut dans l'activité (tableaux 3 et 4), on constate que les différences entre migrants et non-migrants sont négligeables sauf pour la jeune génération des hommes pour laquelle les migrants se retrouvent en plus grand nombre que les non-migrants dans des secteurs rémunérateurs (e.g. comme indépandants et salariés). Par contre, la répartition par secteurs informel et formel (tableau 5) indique que les hommes migrants sont davantage dans le secteur formel que les non-migrants alors que pour les femmes il n'y a pas de différences. Bref, il semble que pour les hommes du moins, le statut migratoire favorise les migrants sur le marché du travail. Il est par ailleurs remarquable que les jeunes générations, quel que soit le statut migratoire ou le sexe, sont nettement défavorisées (e.g. moins d'indépendants et de salariés du public). Toutefois, l'interprétation des différences par génération avec des données transversales sont hasardeuses dans la mesure où les jeunes générations ont eu moins de temps pour accéder aux emplois mieux rémunérés. Il faut attendre l'analyse longitudinale effectuée plus loin pour conclure sur cette question.

Tableau 3 : Répartition de la population migrante de Ouagadougou au moment de l'enquête selon le statut dans l'activité principale, la génération et le sexe

\begin{tabular}{lrrrrrrr}
\hline \multirow{2}{*}{ Génération } & \multicolumn{7}{c}{ Statut dans l'activité principale } \\
\cline { 2 - 8 } & Indépendants & $\begin{array}{r}\text { Salariés } \\
\text { du public }\end{array}$ & $\begin{array}{c}\text { Salariés } \\
\text { du privé }\end{array}$ & Apprentis & $\begin{array}{r}\text { Aide } \\
\text { familial }\end{array}$ & Total & Effectif \\
\hline Hommes & & & & & & & \\
$1936-1955$ & 54,9 & 18,48 & 25,48 & 0,61 & 0,52 & 100 & 204 \\
$1956-1965$ & 47,19 & 27,31 & 23,47 & 1,19 & 0,84 & 100 & 254 \\
$1966-1975$ & 41,81 & 7,89 & 37,28 & 6,37 & 6,64 & 100 & 302 \\
$1976-1985$ & 22,81 & 2,42 & 31,93 & 25,49 & 17,35 & 100 & 77 \\
Femmes & & & & & & & \\
$1936-1955$ & 80,97 & 4,03 & 4,65 & 0 & 10,35 & 100 & 138 \\
$1956-1965$ & 75,19 & 8,98 & 10,05 & 0,61 & 5,18 & 100 & 176 \\
$1966-1975$ & 73,29 & 5,88 & 10,6 & 2,08 & 8,15 & 100 & 231 \\
$1976-1985$ & 37,64 & 1,78 & 22,21 & 2,34 & 36,04 & 100 & 115 \\
\hline
\end{tabular}


Tableau 4 : Répartition de la population non-migrante de Ouagadougou au moment de l'enquête selon le statut dans l'activité principale, la génération, et le sexe

\begin{tabular}{|c|c|c|c|c|c|c|c|}
\hline \multirow[t]{2}{*}{ Génération } & \multicolumn{7}{|c|}{ Statut dans l'activité principale } \\
\hline & Indépendants & $\begin{array}{r}\text { Salariés } \\
\text { du public } \\
\end{array}$ & $\begin{array}{l}\text { Salariés } \\
\text { du privé }\end{array}$ & Apprentis & $\begin{array}{r}\text { Aide } \\
\text { familial } \\
\end{array}$ & Total & Effectif \\
\hline \multicolumn{8}{|l|}{ Hommes } \\
\hline 1936-1955 & 64,99 & 14,3 & 20,7 & 0 & 0 & 100 & 19 \\
\hline 1956-1965 & 55,57 & 10,85 & 23,8 & 1,81 & 7,96 & 100 & 51 \\
\hline 1966-1975 & 45,63 & 4,82 & 34,95 & 6,23 & 8,37 & 100 & 116 \\
\hline 1976-1985 & 28,47 & 0,57 & 12,31 & 38,44 & 20,21 & 100 & 121 \\
\hline \multicolumn{8}{|l|}{ Femmes } \\
\hline 1936-1955 & 96,97 & 0 & 3,03 & 0 & 0 & 100 & 32 \\
\hline $1956-1965$ & 73,85 & 6,56 & 13,33 & 1,98 & 4,28 & 100 & 72 \\
\hline $1966-1975$ & 78,62 & 3,1 & 8,86 & 2,02 & 7,4 & 100 & 137 \\
\hline 1976-1985 & 32,8 & 1,47 & 25,6 & 8,24 & 31,88 & 100 & 121 \\
\hline
\end{tabular}

Tableau 5 : Répartition de la population de Ouagadougou au moment de l'enquête selon le secteur d'activité principale, la génération, le statut migratoire et le sexe

\begin{tabular}{lcccccrrr}
\hline Génération & \multicolumn{4}{c}{ Migrants } & \multicolumn{5}{c}{ Non migrants } \\
\cline { 2 - 9 } & Informel & Formel & Total & Effectif & Informel & Formel & Total & Effectif \\
\hline Hommes & & & & & & & & \\
$1936-1955$ & 66,05 & 33,95 & 100 & 204 & 74,31 & 25,69 & 100 & 19 \\
$1956-1965$ & 62,3 & 37,7 & 100 & 254 & 75,58 & 24,42 & 100 & 51 \\
$1966-1975$ & 75,62 & 24,38 & 100 & 302 & 89,7 & 10,3 & 100 & 116 \\
$1976-1985$ & 95,87 & 4,13 & 100 & 77 & 97,62 & 2,38 & 100 & 121 \\
Femmes & & & & & & & & \\
$1936-1955$ & 92,5 & 7,5 & 100 & 138 & 96,97 & 3,03 & 100 & 32 \\
$1956-1965$ & 85,43 & 14,57 & 100 & 176 & 89,05 & 10,95 & 100 & 72 \\
$1966-1975$ & 88,8 & 11,2 & 100 & 231 & 91,68 & 8,32 & 100 & 137 \\
$1976-1985$ & 99,11 & 0,89 & 100 & 115 & 96,9 & 3,1 & 100 & 121 \\
\hline
\end{tabular}

Soulignons que les données présentées jusqu'ici sont de nature transversale et donnent une idée de la distribution des emplois au moment de l'enquête. Par contre, elles sont muettes sur les processus qui amènent tel groupe à accéder à un emploi donné par rapport à un autre groupe et ne permettent donc pas une véritable analyse des liens entre migration et emploi. L'analyse rétrospective du premier emploi demeure une voie privilégiée à ce titre : les chances d'accès au premier emploi, de même que la nature du premier emploi occupé, demeurent de puissants déterminants de l'avenir. C'est cette voie que nous explorons dans les sections qui suivent. 


\section{Accès au premier emploi}

Nous traitons de l'insertion différentielle des migrants, d'abord de façon statique en considérant les différents statuts occupés lors du premier emploi, puis, en incluant le facteur temps (i.e. la rapidité avec laquelle le premier emploi est obtenu). Gardons également à l'esprit que, contrairement au nonmigrants, les migrants constituent une main-d'œuvre très hétérogène, puisque pour une fraction importante (entre 30 et $45 \%$, tableau 6) il ne s'agit pas de leur premier emploi. Néanmoins, ce qui nous intéresse ici c'est la performance sur le marché du travail de Ouagadougou. Nous reprenons ici deux des trois indicateurs retenus pour l'analyse de la situation au moment de l'enquête, soit le statut dans l'emploi et le secteur d'activité.

Les tableaux 7, 8 et 9 présentent des données descriptives concernant le statut dans l'emploi et le secteur d'activité en fonction du statut migratoire. Pour les hommes, sauf pour la plus vieille génération, les migrants sont davantage dans des statuts non rémunérés (apprenti et aide familial) et dans le statut d'indépendant (tableaux 7 et 8). Pour les femmes, le statut migratoire joue peu. Par contre, les hommes migrants des deux générations plus jeunes se retrouvent légèrement plus que les migrants dans le secteur formel (tableau 9). Ici aussi le statut migratoire joue peu pour les femmes.

Tableau 6 : Migrants selon le Nombre d'emplois exercés avant le premier emploi à Ouagadougou

\begin{tabular}{lccccc}
\hline \multirow{2}{*}{ Statut migratoire } & \multicolumn{6}{c}{ Nombre d'emplois exercés avant le premier emploi à } \\
& \multicolumn{6}{c}{ Ouagadougou } \\
\cline { 2 - 7 } & $\mathbf{0}$ & $\mathbf{1}$ & 2 et plus & Total Effectif \\
\hline Non-migrants & 100 & 0 & 0 & 100 & 517 \\
Rural-Ouagadougou & 46,32 & 31,36 & 22,32 & 100 & 813 \\
Urbain Ouagadougou & 47,95 & 22,63 & 29,42 & 100 & 401 \\
Etranger-Ouagadougou & 32,25 & 28,01 & 39,74 & 100 & 300 \\
\hline
\end{tabular}

Tableau 7 : Répartition de la population migrante occupée dans un premier emploi selon le statut dans l'activité principale, la génération et le sexe

\begin{tabular}{lrrrrrrr}
\hline Génération & Indépendants & $\begin{array}{r}\text { Salarié } \\
\text { du } \\
\text { service } \\
\text { public }\end{array}$ & $\begin{array}{r}\text { Salariés } \\
\text { du } \\
\text { privé }\end{array}$ & Apprentis & $\begin{array}{r}\text { Aide } \\
\text { familial }\end{array}$ & Total & Effectif \\
\hline Hommes & & & & & & & \\
$1936-1955$ & 24,41 & 13,67 & 17,48 & 5,34 & 39,1 & 100 & 254 \\
$1956-1965$ & 13,08 & 20,52 & 20,44 & 9,44 & 36,51 & 100 & 274 \\
$1966-1975$ & 13,53 & 12,94 & 19,39 & 16,13 & 38,01 & 100 & 321 \\
$1976-1985$ & 11,07 & 1,21 & 15,05 & 19,42 & 53,25 & 100 & 81 \\
Femmes & & & & & & & \\
$1936-1955$ & 44,55 & 2,61 & 4,82 & 0,93 & 47,09 & 100 & 162 \\
$1956-1965$ & 37,17 & 8,69 & 7,41 & 2,83 & 43,9 & 100 & 201 \\
$1966-1975$ & 30,95 & 4,55 & 11,12 & 5,97 & 47,41 & 100 & 273 \\
$1976-1985$ & 21,29 & 1,18 & 14,93 & 5,74 & 56,86 & 100 & 145 \\
\hline
\end{tabular}


Une autre approche descriptive de l'analyse du premier emploi est d'estimer la vitesse d'accès à un premier emploi (ici rémunéré) en tenant compte du statut migratoire, du sexe et de la génération (voir la série des courbes Kaplan-Meier : graphique 1 à 6) qui suivent). Le temps n'étant pas défini de la même façon selon le statut migratoire, on ne peut pas mettre les courbes sur le même graphique. Comme nous l'avons mentionné plus haut, pour les non-migrants le temps commence à 12 ans alors que pour les migrants le temps commence au moment de leur arrivée à Ouagadougou. Néanmoins, en examinant les deux premiers graphiques, on peut en déduire que les migrants accèdent plus rapidement que les non migrants à leur premier emploi à Ouagadougou. Cela va dans le même sens que les résultats antérieurs obtenus pour Bamako et Dakar (Piché et Gingras, 1998). Par contre, un résultat qui est très différent des enquêtes antérieures est le peu de différence entre les hommes et les femmes, surtout chez les migrants. Ceci reflète bien le résultat de la stratégie de collecte qui a mis l'accent sur une mesure plus approfondie de l'emploi féminin. L'analyse par génération confirme également les résultats antérieurs à savoir que, quel que soit le statut migratoire ou le sexe, la jeune génération accède plus tardivement (statistiquement significative) à un premier emploi rémunéré que les autres générations (graphiques 3 à 6).

Tableau 8 : Répartition de la population non-migrante occupée dans un premier emploi selon le statut dans l'activité principale, la génération et le sexe

\begin{tabular}{lccccccc}
\hline \multirow{2}{*}{ Génération } & \multicolumn{7}{c}{ Statut dans la première activité principale } \\
\cline { 2 - 8 } & Indépendants & $\begin{array}{c}\text { salariés } \\
\text { du public }\end{array}$ & $\begin{array}{c}\text { Salariés } \\
\text { du privé }\end{array}$ & $\begin{array}{c}\text { Aide } \\
\text { Apprentis } \\
\text { familial }\end{array}$ & Total & Effectif \\
\hline Hommes & & & & & & & \\
$1936-1955$ & 8,77 & 8,49 & 24,06 & 19,64 & 39,04 & 100 & 53 \\
$1956-1965$ & 20,16 & 13,16 & 27,51 & 14,9 & 24,27 & 100 & 91 \\
$1966-1975$ & 24,41 & 5,52 & 19,68 & 26,65 & 23,74 & 100 & 168 \\
$1976-1985$ & 22,17 & 1,24 & 8,81 & 42,35 & 25,44 & 100 & 122 \\
Femmes & 15,7 & 2,9 & 3,38 & 18,88 & 59,14 & 100 & 64 \\
$1936-1955$ & 40,45 & 2,27 & 9,07 & 11,48 & 36,72 & 100 & 109 \\
$1956-1965$ & 32,59 & 2,66 & 7,62 & 9,56 & 47,57 & 100 & 195 \\
$1966-1975$ & 18,69 & 0,49 & 13,92 & 9,8 & 57,1 & 100 & 146 \\
$1976-1985$ & & & & & & & \\
\hline
\end{tabular}


Tableau 9 : Répartition de la population occupée dans un premier emploi selon le secteur D'activité, la génération, le sexe et le statut migratoire

\begin{tabular}{lrrrrrrrr}
\hline Génération & \multicolumn{4}{c}{ Migrants } & \multicolumn{5}{c}{ Non-migrants } \\
\cline { 2 - 9 } & Informel & Formel & Total & Effectif & Informel & Formel & Total & Effectif \\
\hline Hommes & & & & & & & & \\
$1936-1955$ & 81,06 & 18,94 & 100 & 249 & 83,37 & 16,63 & 100 & 53 \\
$1956-1965$ & 76,9 & 23,1 & 100 & 266 & 89,07 & 10,93 & 100 & 91 \\
$1966-1975$ & 79,79 & 20,21 & 100 & 319 & 91,86 & 8,14 & 100 & 168 \\
1976-1985 & 91,18 & 8,82 & 100 & 80 & 99,34 & 0,66 & 100 & 122 \\
Total & 80,77 & 19,23 & 100 & 914 & 93,21 & 6,79 & 100 & 434 \\
Femmes & & & & & & & & \\
1936-1955 & 93,88 & 6,12 & 100 & 162 & 91,84 & 8,16 & 100 & 64 \\
1956-1965 & 84,64 & 15,36 & 100 & 200 & 92,18 & 7,82 & 100 & 109 \\
$1966-1975$ & 94,43 & 5,57 & 100 & 273 & 96,18 & 3,82 & 100 & 195 \\
1976-1985 & 99,41 & 0,59 & 100 & 145 & 99,02 & 0,98 & 100 & 146 \\
Total & 93,47 & 6,53 & 100 & 780 & 96,22 & 3,78 & 100 & 514 \\
\hline
\end{tabular}

\section{Les déterminants de l'accès à un premier emploi rémunéré}

Afin de bien comprendre le lien entre migration et emploi, nous privilégions ici le premier emploi rémunéré (notre variable dépendante). Les travaux récents sur l'insertion économique des migrants insistent sur un certain nombre de facteurs clé tant au niveau macro que micro (Piché, 2004). La variable clé au niveau macro est l'état du marché du travail: ici, nous utilisons la génération comme indicateur («proxy») de cet état. En effet, chaque génération a connu des contextes différents, reflétant la situation du marché, et par conséquent au même âge, leurs conditions de vie peuvent ne pas être identiques. Au niveau micro, quatre déterminants importants sont reliés au capital humain (l'éducation), au genre (sexe), à l'origine sociale (activité du père et de la mère) et enfin à l'origine ethnique (voir tableau 10). Le modèle 1 du tableau 10 montre l'effet du statut migratoire en contrôlant pour la génération: il confirme ce qui avait été trouvé avec les tableaux croisés, à savoir que les migrants accèdent plus rapidement à leur premier emploi à Ouagadougou que les non migrants et que la jeune génération prend beaucoup plus de temps à accéder à son premier emploi rémunéré que les générations précédentes. Le modèle 2 introduit la variable sexe et confirme ce qui est maintenant largement documenté, à savoir l'avantage qu'ont les hommes sur les femmes du moins en ce qui concerne à l'emploi rémunéré.

Le dernier modèle du tableau 10 introduit les autres variables (éducation, origines sociale et ethnique). D'abord, on voit que l'introduction de ces variables ne changent pas l'effet net du statut migratoire : les migrants, quel que soit leur origine, accède plus facilement à un premier emploi que les non migrants. Il est intéressant de noter que c'est le fait d'avoir effectué une migration rurale-urbaine qui augmente le plus les chances d'accéder à un premier emploi rémunéré. Quant au rôle de l'éducation, on constate que les 
non instruits accèdent plus rapidement à l'emploi rémunéré que les autres catégories d'instruits. Ce résultat peut sembler contradictoire avec la théorie $\mathrm{du}$ capital humain, mais en fait, dans une approche longitudinale, l'éducation a comme effet de retarder l'accès à l'emploi. Les non instruits accèdent ainsi plus vite à l'emploi pendant que les autres vont à l'école. Des analyses plus approfondies (en cours) montrent que les non instruits se retrouvent davantage dans des petits emplois de commerce par exemple et que les instruits accèdent davantage au secteur formel. S'agissant de l'origine sociale mesurée par l'activité du père, elle n'est pas significative alors qu'elle l'est lorsque mesurée par l'activité de la mère. En effet, les chances d'accès à un premier emploi rémunéré diminuent de façon significative lorsque la mère est salariée (versus mère indépendante). Peutêtre peut-on voir là un indicateur du caractère familial du secteur informel et l'effet d'une stratégie familiale où les mères travaillant comme indépendantes dans le secteur informel font davantage appel au travail de leurs enfants. Enfin, l'origine ethnique semble jouer très peu : seul l'ethnie «bobo» semble avoir un avantage sur l'ethnie «mossi». Ce résultat méritera d'être approfondi par des analyses plus qualitatives.

\section{Conclusion et discussion}

Quel est le rôle de la migration sur l'accès à l'emploi ? Les analyses présentées ici suggèrent quatre conclusions importantes. Premièrement, le rôle de la migration sur l'accès à l'emploi joue très peu pour les femmes. Quel que soit leur statut migratoire, celles-ci sont toujours désavantagées sur le marché de l'emploi rémunéré: elles sont confinées dans le secteur informel comme indépendantes (essentiellement le petit commerce). De plus, le fait qu'il s'agisse d'une sphère où le travail familial est important pourrait expliquer l'effet de l'activité indépendante de la mère sur les chances d'accès plus rapide à un premier emploi rémunéré, lui aussi probablement appartenant à la même sphère. Cela confirme la nécessité de théories migratoires spécifiques pour les hommes et les femmes.

La deuxième conclusion sur le rôle de la migration concerne les hommes. Pour ces derniers, les données au moment de l'enquête semblent indiquer un léger avantage des migrants sur les non migrants, avantage mesuré ici par un plus faible taux de chômage et des proportions plus élevées dans des emplois rémunérés et dans le secteur formel. Les analyses multivariées effectuées sur le premier emploi rémunéré confirment l'avantage "net » des migrants sur les non-migrants. Pour expliquer ce résultat, certains auteurs avancent l'hypothèse que les migrants n'ont pas le choix de rester longtemps en situation d'inactivité et doivent donc accepter n'importe quel emploi (Obérai et Singh, 1984) ou alors retourner en milieu rural, laissant dans la ville ceux qui auraient « réussi » leur insertion économique (Pott, 2000). Ces deux hypothèses ne semblent pas rendre compte de la dynamique migration-emploi dans le cas de Ouagadougou. Dans le premier cas, il faut noter que les migrants accèdent également plus rapidement au secteur 
formel (Bocquier et Le Grand, 1998). ${ }^{7}$ Dans le deuxième cas, l'enquête étant nationale, nous avons pu tenir compte de l'ensemble de la population ayant séjourné au moins une fois à Ouagadougou. Ainsi malgré la présence des absents de Ouagadougou dans notre population «exposée aux risques", le statut migratoire demeure un déterminant puissant de l'accès au premier emploi rémunéré. Bref, les théories migratoires qui considèrent les migrants comme venant rejoindre les marginaux et les chômeurs urbains doivent être complètement revues. Deux facteurs peuvent expliquer l'effet positif de la migration : la sélectivité migratoire et le système d'information propre aux migrants. Le premier facteur est plus connu et avancé surtout par les économistes : ceux qui migrent seraient les plus dynamiques. Le deuxième facteur, relié à la théorie de la recherche d'emploi, est moins documenté pour les pays en développement mais est au cœur des théories du marché du travail dans les pays développés (Granovetter, 2000). Ainsi, le migrant potentiel est tenu au courant de l'état du marché du travail, soit par ses réseaux familiaux et d'amis, soit par des visites de courte durée sur son futur lieu d'embauche. Il ne viendrait à la ville que s'il sait qu'il a de fortes chances de trouver un emploi.

La troisième conclusion a trait au rôle de l'origine ethnique. Dans la littérature sur les pays développés, les inégalités ethniques sur le marché du travail sont largement documentés (Reitz, 1997) et constituent une pièce maîtresse des théories de l'immigration (Piché, 2004). Les résultats présentés ici montre le peu d'impact de l'origine ethnique sur l'accès à l'emploi. Cela ne veut pas dire que les réseaux ethniques ne sont pas à l'œuvre (au contraire nous y faisons référence ci-haut) mais que le marché du travail ne serait pas structuré sur une base ethnique.

Enfin, la dernière conclusion liée aux effets de génération nous permet de revenir, en terminant, à la question de la pauvreté. Si la migration continue à jouer un rôle positif sur le marché du travail en l'an 2000 à Ouagadougou, son effet n'élimine pas l'effet de génération. En effet, la jeune génération née entre 1976 et 1985, et qui entre sur le marché du travail au cours de la période 1990-2000, se trouve nettement défavorisée par rapport aux générations précédentes. Rappelons la diminution drastique de la proportion des travailleurs salariés du public dans cette génération, indépendamment du sexe ou du statut migratoire. C'est le groupe de population qui commence son entrée dans la vie active à la fin des années 1980. Or, depuis le début des années 1980, le pays, affecté par des déficits budgétaires grandissants et une récession économique persistante, continu le gel des embauches accompagné de réduction des effectifs dans le secteur public suite à l'adoption de programmes d'ajustement structurel. On pourrait d'ailleurs se demander si ce n'est pas la limite d'emploi du secteur public, source de maintien hors de la pauvreté, qui est à l'origine de l'augmentation de la pauvreté à Ouagadougou.

7 Nos analyses en cours sur Ouagadougou confirment également l'accès plus rapide des migrants au secteur formel. 


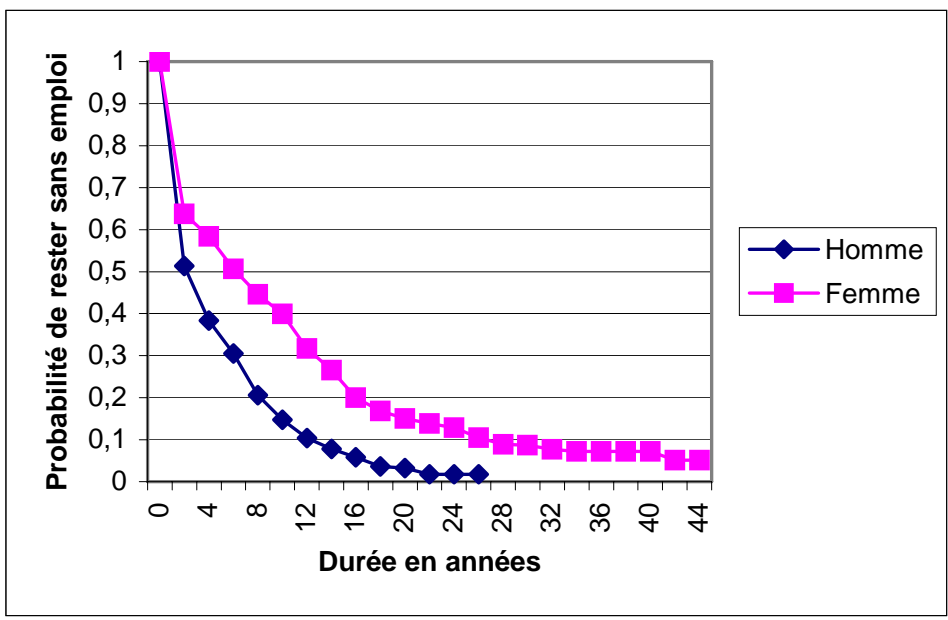

Graphique 1 : Temps mis pour accéder au premier emploi rémunéré chez les migrants selon le sexe

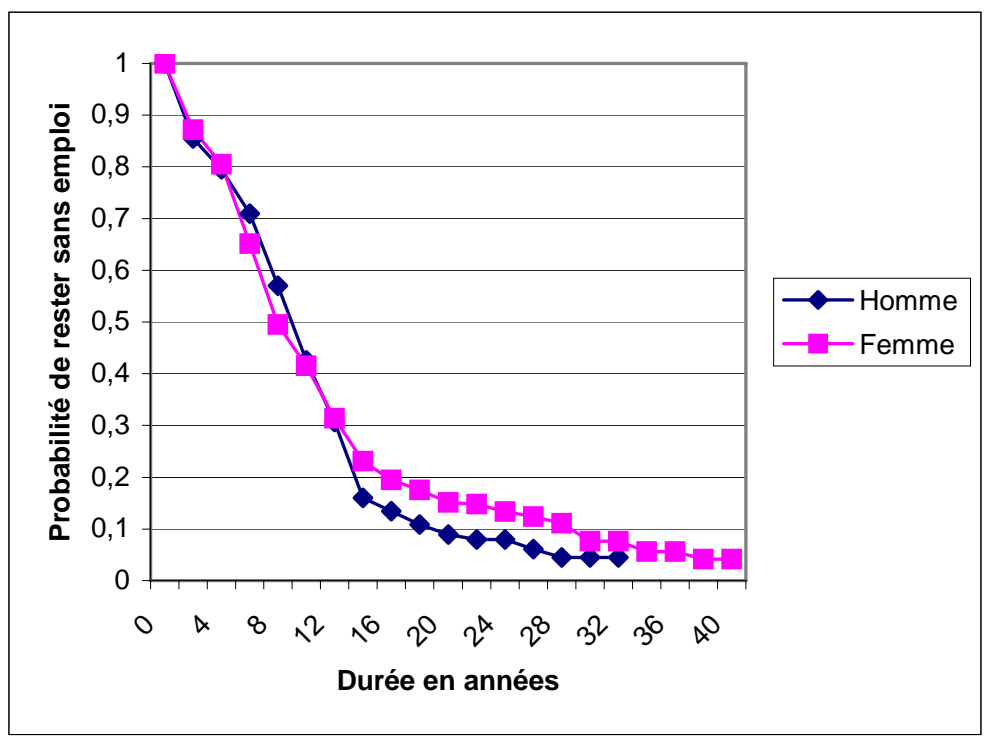

Graphique 2: Temps mis pour accéder à un premier emploi rémunéré chez les non-migrants selon le sexe 


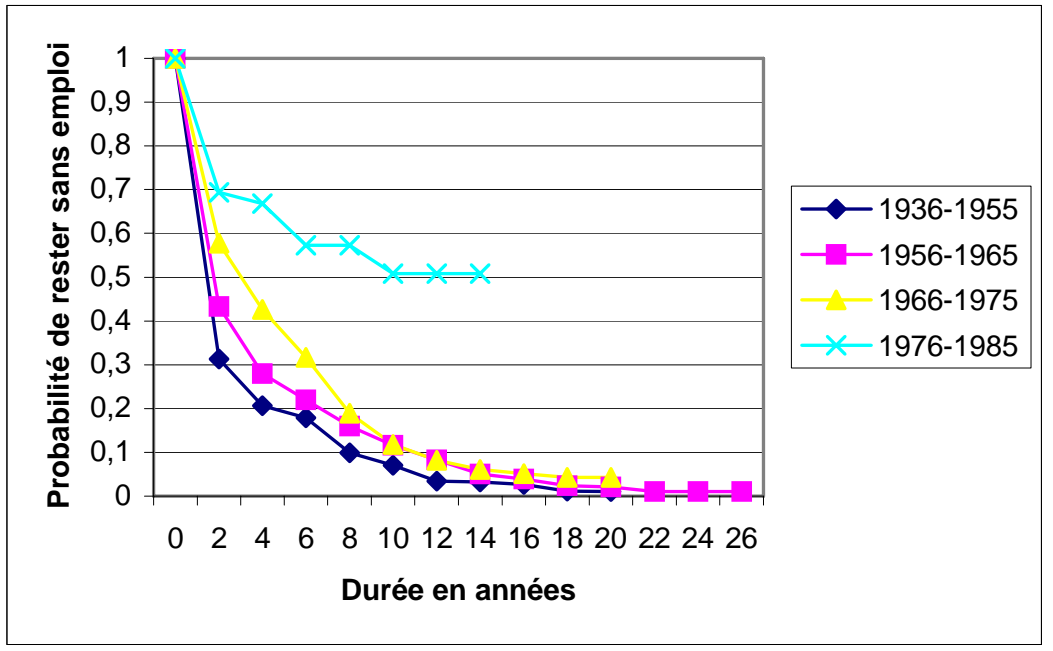

Graphique 3 : Temps mis pour accéder au premier emploi rémunéré chez les hommes migrants selon la génération

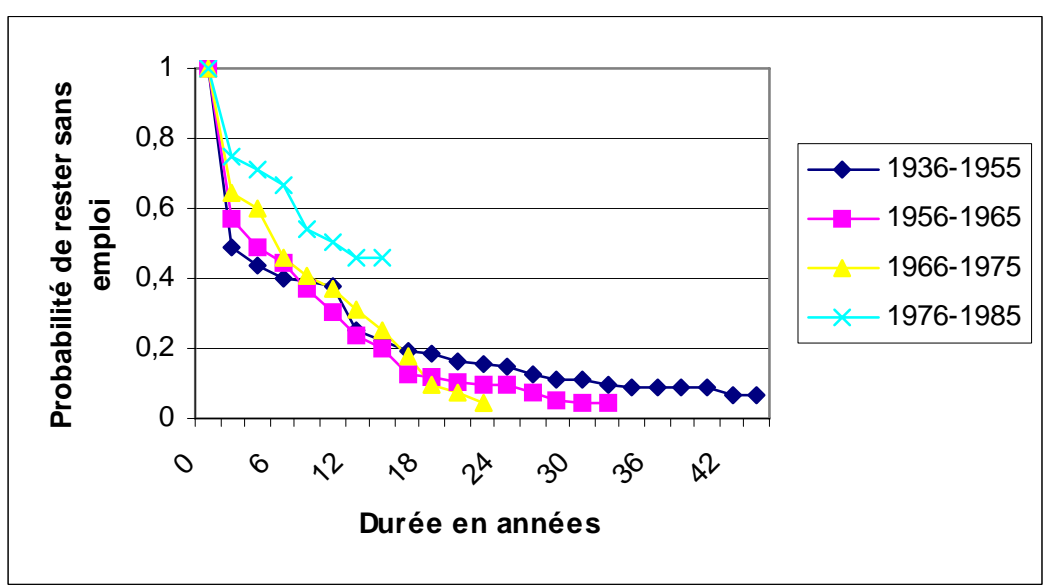

Graphique 4 : Temps mis pour accéder au premier emploi rémunéré chez les femmes migrantes selon la génération 


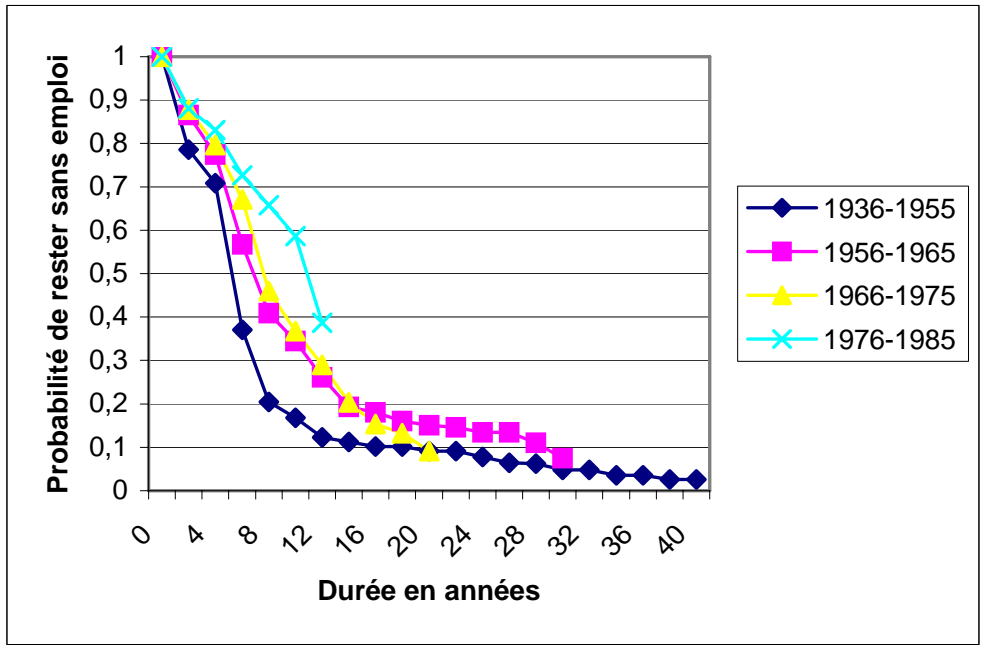

Graphique 5 : Temps mis pour accéder au premier emploi rémunéré chez les non-migrantes selon la génération

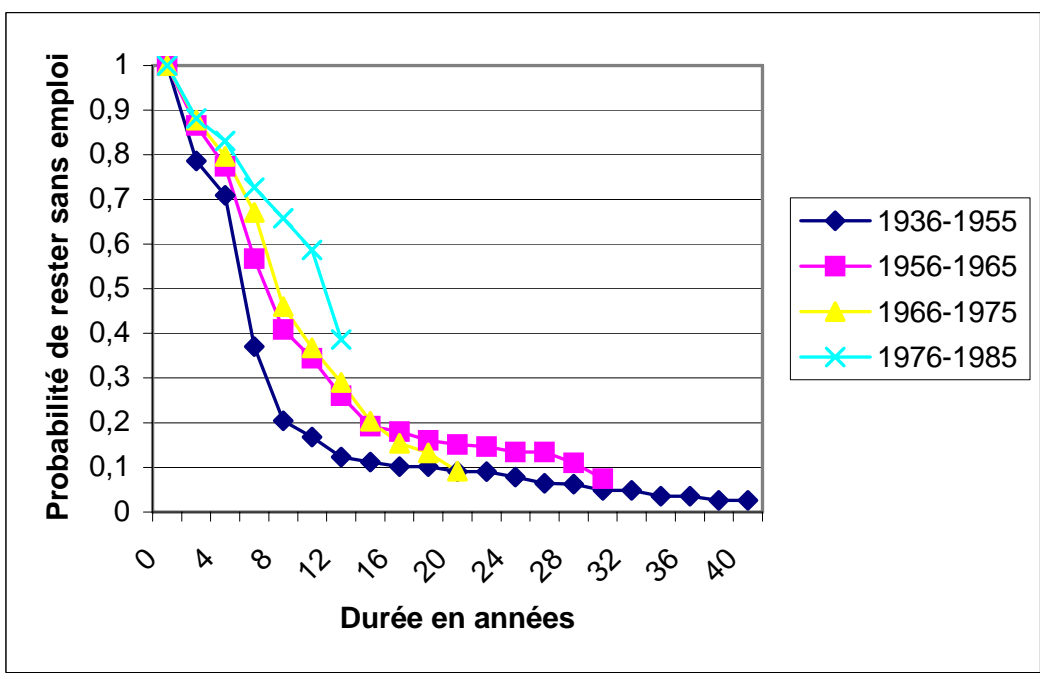

Graphique 6 : Temps mis pour accéder au premier emploi chez les nonmigrantes selon la génération 
http://aps.journals.ac.za

Zourkaléini Younoussi EVictor Piché : Migration et emploi urbain

Tableau 10: Résultats des régressions de Cox: l'accès à un premier emploi rémunéré a Ouagadougou

\begin{tabular}{|c|c|c|c|c|c|c|c|}
\hline \multirow{2}{*}{$\begin{array}{l}\text { Catégorie de } \\
\text { référence }\end{array}$} & \multirow[t]{2}{*}{ Variables } & \multicolumn{2}{|c|}{ Modèle 1} & \multicolumn{2}{|c|}{ Modèle 2} & \multicolumn{2}{|c|}{ Modèle 3} \\
\hline & & Coefficient & $P>|z| C$ & efficientI & $>|z| C$ & CoefficientP & $>|z|$ \\
\hline \multirow{3}{*}{ Non-migrants } & Rural $\longrightarrow$ Ouaga & 1,87 & 0,00 & 1,77 & 0,00 & 1,73 & $\overline{0,00}$ \\
\hline & Urbain $\longrightarrow$ Ouaga & 1,37 & 0,00 & 1,60 & 0,00 & 1,53 & 0,00 \\
\hline & Etranger $\longrightarrow$ Ouaga & 1,46 & 0,00 & 1,48 & 0,00 & 1,43 & 0,00 \\
\hline \multirow{5}{*}{ génération 76-85 } & Généraion & & & & & & \\
\hline & Generation: $<=55$ & 2,74 & 0,00 & 2,35 & 0,00 & 2,32 & 0,00 \\
\hline & Generation:56-65 & 2,62 & 0,00 & 2,32 & 0,00 & 2,28 & 0,00 \\
\hline & Generation:66-75 & 2,17 & 0,00 & 2,01 & 0,00 & 1,99 & 0,00 \\
\hline & Sexe & & & & & & \\
\hline \multirow[t]{5}{*}{ Femme } & Masculin & & & 1,67 & 0,00 & 1,69 & 0,00 \\
\hline & Niveau d'éducation att & vant le premie & er emploi & & & & \\
\hline & Médersa & & & 0,63 & 0,00 & 0,63 & 0,00 \\
\hline & Primaire & & & 0,91 & 0,34 & 0,92 & 0,40 \\
\hline & sec, Profe & & & 0,58 & 0,00 & 0,60 & 0,00 \\
\hline \multirow[t]{8}{*}{ Sans éducation } & Sec, 1cycle & & & 0,55 & 0,00 & 0,56 & 0,00 \\
\hline & sec, 2cycle & & & 0,51 & 0,00 & 0,53 & 0,00 \\
\hline & Supérieur & & & 0,48 & 0,00 & 0,49 & 0,00 \\
\hline & Statut du père dans sor & ité principale & & & & & \\
\hline & Salarié dans le public & & & & & 0,94 & 0,48 \\
\hline & Salarié dans le privé & & & & & 1,13 & 0,35 \\
\hline & Autre & & & & & 1,48 & 0,28 \\
\hline & Statut de la mère dans & tivité princip & ale & & & & \\
\hline \multirow[t]{4}{*}{ Indépendant } & salarié dans le public & & & & & 0,64 & 0,02 \\
\hline & Salarié dans le privé & & & & & 0,58 & 0,05 \\
\hline & Aide familiale & & & & & 1,06 & 0,53 \\
\hline & Autres & & & & & 0,86 & 0,07 \\
\hline \multirow[t]{5}{*}{ Indépendant } & Ethnie & & & & & & \\
\hline & Bobo & & & & & 1,52 & 0,02 \\
\hline & Peul & & & & & 1,11 & 0,55 \\
\hline & Dagara & & & & & 1,05 & 0,85 \\
\hline & Senoufo & & & & & 1,16 & 0,34 \\
\hline \multirow[t]{5}{*}{ Mossi } & Gourounsi & & & & & 1,06 & 0,66 \\
\hline & Bissa & & & & & 1,22 & 0,47 \\
\hline & Gourmantché & & & & & 1,03 & 0,90 \\
\hline & Lobi & & & & & 1,23 & 0,38 \\
\hline & Autres ethnies & & & & & 1,35 & 0,02 \\
\hline
\end{tabular}




\section{Bibliographie}

Adepoju A. 1988. "Migration et urbanisation en Afrique : problèmes et politiques ", dans Van de Walle E., Sala-Diakanda D. et Ohadike P. (éds), L'état de la démographie africaine, Liège (Belgique), Union Internationale pour l'Étude Scientifique de la Population, p. 123-138.

Antoine P. et Piché V. 1998. «L'insertion à Dakar et à Bamako : une comparaison des itinéraires », dans Antoine P., Ouédraogo D. et Piché V. (éds), Trois générations de citadins au Sahel. Trente ans d'histoire sociale à Dakar et à Bamako, Paris, L'Harmattan, p. 11-44.

Beauchemin C. 2001. L'émergence de l'émigration urbaine en Côte d'Ivoire, Paris et Abidjan, CEPED et INS, Les Études du CEPED no 19.

Bocquier P. et LeGrand T. 1998. "L'accès à l'emploi dans le secteur moderne », dans Antoine P., Ouédraogo D. et Piché V. (éds), Trois générations de citadins au Sahel. Trente ans d'histoire sociale à Dakar et à Bamako, Paris, L'Harmattan, p. 77-116.

Burkina Faso. 2000. Cadre stratégique de lutte contre la pauvreté,Ouagadougou, Ministère de l'Économie et des Finances.

Granovetter M. 2000. Le marché autrement, Paris, Desclée de Brouwer.

ILO. 1997. Jobs for Africa: A Policy Framework for an Employment-Intensive Growth Stragegy, Geneve, ILO.

INSD. 1999. Analyse de la pauvreté au Burkina Faso

Kishimba N. 2002. "Générations et processus d'insertion sur le marché de l'emploi à Yaoundé ", dans Gendreau, F., Tabutin, D.et Poupard M. (éds), Jeunesses, vieillesses, démographies et sociétés, Louvain-la-Neuve, Academia_bruylant/L'Harmattan, p. 197-218.

Kouamé A. 1991. "Les conditions socio-démographiques de la reproduction du secteur informel et des milieux urbains africains défavorisés: quelques réflexions théoriques à partir du cas ivoirien ", Revue canadienne d'études $d u$ développement, Vol. 12, No 2, p. 405-420.

Kouamé A. 2000. «L'insertion des migrants sur le marché du travail: une revue de literature", Études et Recherches no 2, Dakar, Union for African Population. PA No. 2.

Oberai A.S. et Singh H.K.M. 1984. « Les migrations, l'emploi et le marché du travail urbain : le cas du Pendjab indien ", Revue internationale du travail, Vol. 123, No 4, p. 553-572.

Piché V. 2004. «Immigration et intégration dans les pays développés: un cadre conceptuel », dans Caselli, Graziella, Vallin, Jacques et Wunsch, Guillaume (éds), Démographie: analyse et synthèse. Volume 6: Conséquences des changements démographiques, Paris, INED ; chap. 84.

Piché V., Mariko S. et Gingras L. 1995. «L'emploi et l'insertion sur le marché du travail », dans Ouédraogo D. et Piché V., L'insertion urbaine à Bamako, Paris, Karthala, p. 59-80.

Piché V. et Gingras L. 1998. «Migrer un atout pour l'emploi? », dans Antoine P., Ouédraogo D. et Piché V., Trois générations de citadins au Sahel. Trente ans d'histoire sociale à Dakar et à Bamako, Paris, L'Harmattan, p. 47-76.

Poirier J., Piché, V., Le Jeune G., Dabiré B. et Wane H.R. 2001. « Projet d'étude des stratégies de reproduction des populations sahéliennes à partir de l'enquête Dynamique migratoire, insertion urbaine et environnement au Burkina Faso », Cahiers québécois de démographie, Vol. 30, No 2, p. 289-310.

Portes A. et Shauffer R. 1993. "Competing Perspectives on the Latin American Informal Sector », Population and Development Review, Vol. 19, No 1, p. 33-60.

Potts D. 2000. «Urban Unemployment and Migrants in Africa: Evidence from Harare 1985-1994 », Development and Change, Vol. 31, p. 879-910. 
Reitz J. 1997. «Ethnic Concentration in Labour Markets and Their Implications for Ethnic Stratification », dans Van den Berg A. et Smucker J. (éds), The Sociology of Labour Markets, Scarborough (Ontario, Canada), Prentice Hall Allyn et Bacon Canada, p. 409-450.

Somda P. et Sawadogo S.M. 2001. " Le suivi de la pauvreté au Burkina Faso: instruments et contraintes ", dans Koulibaly M., La pauvreté en Afrique de l'Ouest, Dakar et Paris, CODESRIA et Karthala, p. 89-112.

Todaro M. 1971. «Income Expectations, Rural-Urban Migration and Employment in Africa », International Labour Review, Vol. 104, p. 387-413.

Traoré S. 1997. "L'insertion professionnelle en milieu urbain ouest-africain ", dans Rallu J.-L., Courbage Y. et Piché V. (éds), Old and New Minorities/Anciennes et nouvelles minorités, Paris, John Libbey et INED, p. 251-268. 